Supporting Information for

\title{
Size and Shape Dependence of the Electronic Structure of Gold Nanoclusters on $\mathrm{TiO}_{2}$
}

Chi-Ming Yim ${ }^{\dagger}$ Philomena Schlexer Lamoureux, ${ }^{\dagger}$ Andrew Mellor ${ }^{\dagger}$, Chi L. Pang, ${ }^{\dagger}$ Hicham Idriss ${ }^{\S}$, Gianfranco Pacchioni, $\stackrel{\star}{*}$ and Geoff Thornton ${ }^{\dagger *}$

$\dagger^{\dagger}$ Department of Chemistry and London Centre for Nanotechnology, University College London, 20 Gordon Street, London WC1H 0AJ, UK

$\$$ Dipartimento di Scienza dei Materiali, Università degli Studi Milano-Bicocca, via R. Cozzi, 55, 20126 Milano, Italy

$\S$ Surface Science and Advanced Characterisation, Chemical Sciences Division, SABIC-CRD at KAUST, Thuwal 23955, Saudi Arabia

This PDF file contains:

Experimental Details, Computational Details, Supporting Figures S1-S7. 


\section{Experimental Details}

The STM experiments were performed using an Omicron $\mathrm{GmbH}$ low temperature scanning tunneling microscope housed in an ultrahigh vacuum bath cryostat with a base pressure of $3 \times 10^{-11}$ mbar. The adjoining preparation chamber was equipped with an ion sputter gun and sample heater, as well as facilities for X-ray photoelectron spectroscopy (XPS) and low energy electron diffraction (LEED) measurements. $\quad \mathrm{TiO}_{2}(110)-(1 \times 1)$ single crystal samples $($ PiKem $)$ were prepared through cycles of argon ion sputtering and vacuum annealing at $\sim 1000 \mathrm{~K}$. Sample cleanliness and long-range order were confirmed using XPS and LEED, respectively. Deposition of Au onto the sample was achieved using an evaporation source comprising a high purity gold (Au) wire (Goodfellow, purity $>99.99 \%$ ) wrapped with a tungsten coil, which was resistively heated. The deposition rate of the evaporation source was calibrated using XPS, with the Au coverage on the $\mathrm{TiO}_{2}(110)$ surface being estimated from the STM images. STM topographic images were collected in the constant current mode using electro-chemically etched tungsten tips, conditioned by outgassing at $500 \mathrm{~K}$ and STM voltage pulsing. Point differential conductance $(\mathrm{d} I / \mathrm{d} V)$ spectra were recorded using a standard lock-in technique with the frequency of bias modulation set at $573 \mathrm{~Hz}$. All STM images and STS spectra were collected at $78 \mathrm{~K}$.

\section{Computational Details}

Periodic, spin polarized density functional theory (DFT) calculations have been performed using the Vienna $\mathrm{Ab}$ Initio Simulation Package (VASP 5.2). ${ }^{1-4}$ Generalized gradient approximations (GGA) for the exchange-correlation functional were applied within the Perdew, Burke and Ernzerhof (PBE) formulation. 5,6 To describe electron-ion interactions, the projector augmented wave (PAW) method was used. ${ }^{7,8} \mathrm{O}(2 \mathrm{~s}, 2 \mathrm{p}), \mathrm{Ti}(3 \mathrm{~s}, 4 \mathrm{~s}, 3 \mathrm{p}, 3 \mathrm{~d})$, and $\mathrm{Au}(5 \mathrm{~d}, 6 \mathrm{~s})$ are 
described as valence electrons and were consequently treated explicitly. For electronic relaxations, we used the blocked Davidson iteration scheme. ${ }^{9}$

For transition metal oxides such as $\mathrm{TiO}_{2}$ the GGA approach suffers from the selfinteraction error, which significantly affects the electronic structure. To circumvent this error, we used the GGA+U approach as proposed by Dudarev and co-workers. ${ }^{10}$ With this approach the multiple occupations of $\mathrm{d}$ orbitals is penalized so that the underestimation of the band gap and electron delocalization is attenuated. In this work, we set the U-parameter to $3 \mathrm{eV}$ for the Ti $3 \mathrm{~d}$ states. With these parameters a good qualitative description of electronic and geometric structures is given (see below). The GGA approach in combination with the PBE exchange-correlation functional does not include dispersion forces. Because these forces may be important for the description of the cluster-support interaction, ${ }^{11,12}$ we used the semi-empirical dispersion correction known as the DFT-D2 approach as proposed by Grimme. ${ }^{13}$ It is generally assumed that the DFTD2 method produces an overestimate of the dispersion interactions. For this reason we changed the parameters C6 and R0 of the DFT-D2 approach, as suggested by Tosoni and Sauer. ${ }^{14}$ We denote this method as DFT-D2'.

In geometric structure optimizations, all ions were allowed to relax until ionic forces are smaller than $|0.01| \mathrm{eV} / \AA$. Calculation of the bulk structures were done using a kinetic energy cutoff of $900 \mathrm{eV}$ for $\mathrm{TiO}_{2}$ and a $\Gamma$-centred k-point grid, which was set to $(8 \times 8 \times 8)$. The obtained lattice parameters are $\mathrm{a}_{0}=\mathrm{b}_{0}=4.640 \AA$, and $\mathrm{c}_{0}=3.007 \AA$, which gives a deviation of the cell volume of $3.67 \%$ with respect to experimental findings. ${ }^{15}$ For all subsequent structure investigations, $\Gamma$-point calculations were performed and wave functions were expanded in the plane wave basis up to a kinetic energy of $400 \mathrm{eV}$. 
To investigate the rutile $\mathrm{TiO}_{2}(110)$ surface, we chose a $(3 \times 2)$ surface unit cell with lattice parameters of $\mathrm{a}_{0}$, surf $=9.02 \AA$, and $\mathrm{b}_{0, \text { surf }}=13.13 \AA$. The slab consists of five $\mathrm{TiO}_{2}$ layers and the distance between slabs is larger than $12 \AA$, in order to minimize interactions with the replica. All atoms were allowed to relax during structure optimizations.

Adsorption energies ( $\left.E_{a d}\right)$ were calculated as defined in Equation (1), where all components refer to structure optimized systems.

$$
E_{\text {ad }}\left(\mathrm{Au}_{\mathrm{n}} / \mathrm{TiO}_{2}\right)=E\left(\mathrm{Au}_{\mathrm{n}} / \mathrm{TiO}_{2}\right)-E\left(\mathrm{Au}_{\mathrm{n}}\right)-E\left(\mathrm{TiO}_{2}\right) \quad \text { Equation (1) }
$$

Atomic charges have been estimated with the Bader decomposition scheme. ${ }^{16-18}$ Effective Bader charges are defined as $Q_{\text {eff }}=Z_{V A L}-q$, where $Z_{V A L}$ is the number of valence electrons and $q$ is the Bader charge as given by the Bader analysis. To simulate the tunneling current for STM images, the Tersoff-Hamann method ${ }^{19}$ was used. The sum of the local density of states in the energy range between the Fermi level and the bias potential at the tip position was used. 


\section{Supporting Figures}
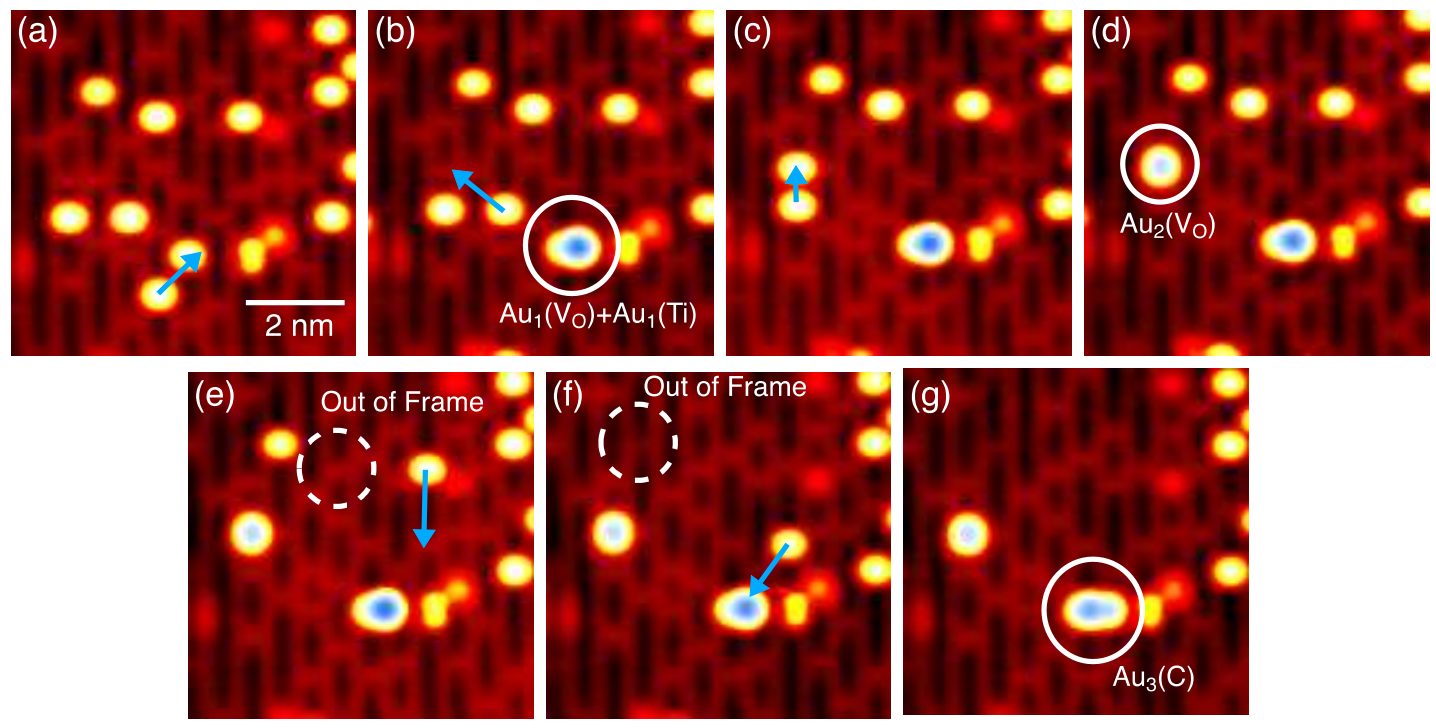

Figure S1. Sequential STM images of $r-\mathrm{TiO}_{2}$ loaded with $\sim 0.01 \mathrm{ML} \mathrm{Au}$, showing the formation of different Au clusters from single Au adatoms by $+3 \mathrm{~V}$ tip pulses. (a)-(g) Shows the formation of: (a),(b) a $\mathrm{Au}_{1}\left(\mathrm{~V}_{\mathrm{O}}\right)+\mathrm{Au}_{1}(\mathrm{Ti})$ complex; (c),(d) $\mathrm{Au}_{2}\left(\mathrm{~V}_{\mathrm{O}}\right)$; (e)-(g) $\mathrm{Au}_{3}(\mathrm{C})$. Arrows indicate the movement of the Au adatoms triggered by voltage pulses. Dashed circles mark the Au atoms that have gone out of the imaged region as a result of voltage pulses. Image size: $(7 \mathrm{~nm})^{2}$. Scan parameters: $0.8 \mathrm{~V}, 0.2 \mathrm{nA}$. 

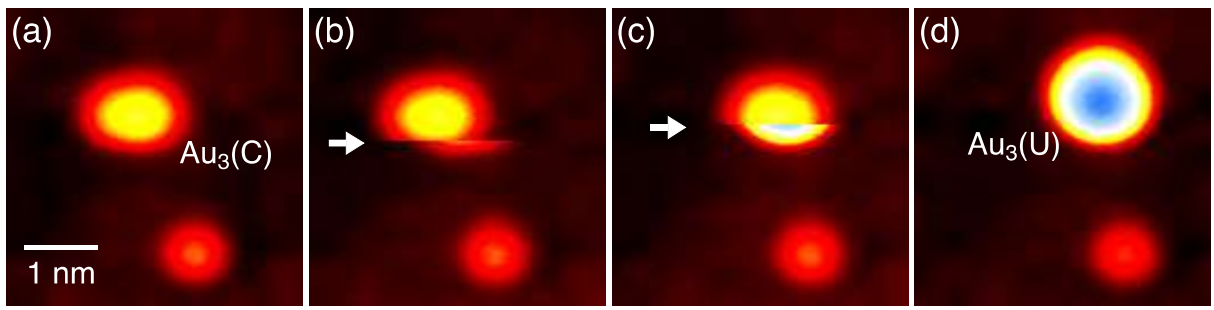

Figure S2. (a-d) Sequential STM images showing how an $\mathrm{Au}_{3}(\mathrm{C})$ cluster is (b) 'flipped' across the $\mathrm{O}_{\mathrm{b}}$ row direction, (c) then 'lifted' by the STM tip during high voltage scan at $+2.4 \mathrm{~V}$, forming an $\mathrm{Au}_{3}(\mathrm{U})$ cluster in (d). Image size: $(4 \mathrm{~nm})^{2}$. Scan parameters: (a-c) $2.4 \mathrm{~V}, 0.1 \mathrm{nA}$; (d) $2.4 \mathrm{~V}, 10$ pA. Arrows indicate the moments of tip-cluster interaction. 

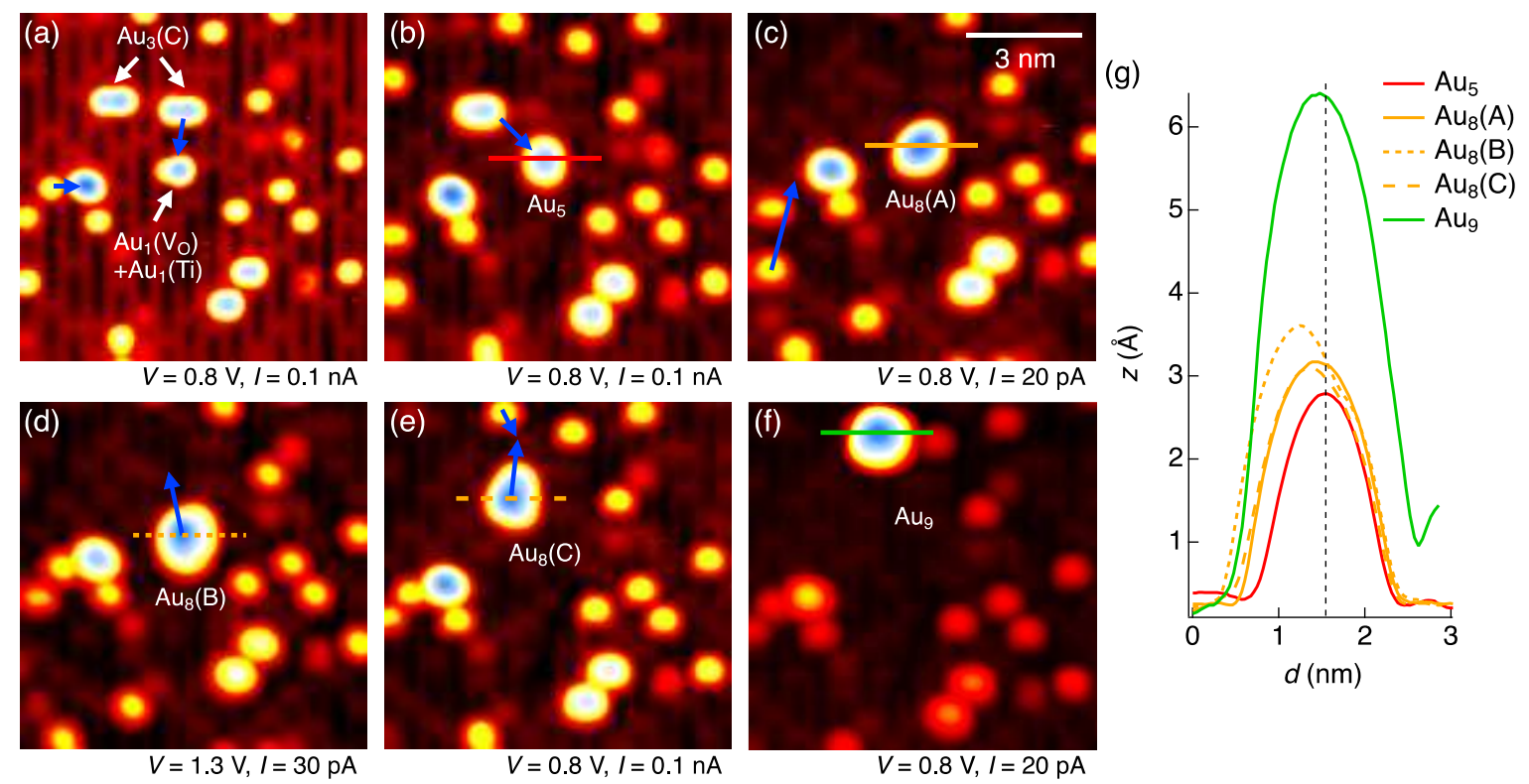

Figure S3. STM topographic images showing the step-by-step formation of different sized Au clusters on $\mathrm{r}-\mathrm{TiO}_{2}$ by $+3 \mathrm{~V}$ voltage pulses. (a)-(f) Shows the formation of: (a),(b) $\mathrm{Au}_{5}$, (c-e) $\mathrm{Au}_{8}$, and (f) $\mathrm{Au}$. . Blue arrows indicate the pulse-induced displacement of the $\mathrm{Au}_{\mathrm{n}}$ species. Image size: $(9 \mathrm{~nm})^{2}$. (g) Line profiles across different $A u_{n}$ species as marked in (b)-(f). 

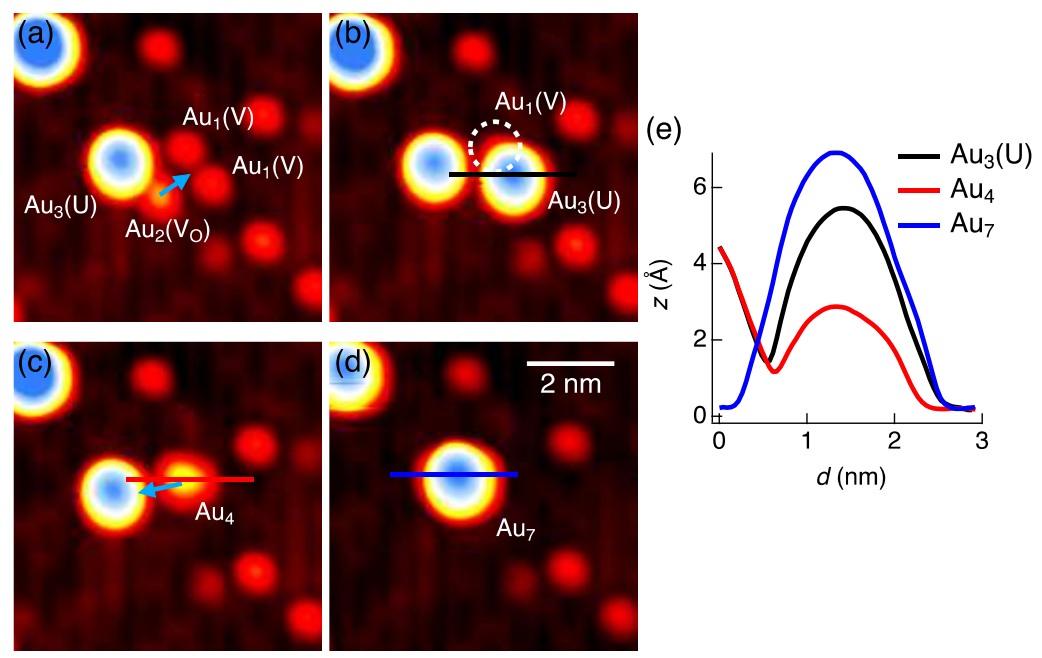

Figure S4. STM images showing the step-by-step formation of different sized Au clusters using $+3 \mathrm{~V}$ voltage pulses in STM. (a)-(d) shows the formation of: (a), (b) an $\mathrm{Au}_{3}(\mathrm{U})$; (c) $\mathrm{Au}_{4}$; (d) $\mathrm{Au}_{7}$ cluster. Image size: $(7 \mathrm{~nm})^{2}$. Scan parameters: $1 \mathrm{~V}, 0.1 \mathrm{nA}$. (g) Line plots taken across the different $\mathrm{Au}$ clusters marked in (b-d), showing that the Au $\mathrm{Au}_{4}$ cluster in (c) has a flat morphology thus a lower height compared to $\mathrm{Au}_{3}(\mathrm{U})$, while the $\mathrm{Au}_{7}$ cluster is the tallest $(\sim 650 \mathrm{pm})$. 


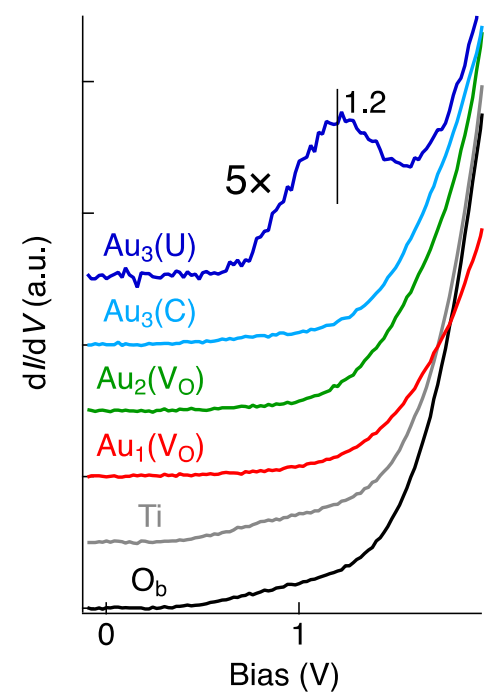

Figure S5. Constant height $\mathrm{d} I / \mathrm{d} V$ spectra recorded from the $\mathrm{TiO}_{2}(110)$ substrate (black: $\mathrm{O}_{\mathrm{b}}$, grey: $\mathrm{Ti}_{5 \mathrm{c}}$ ), as well as from different $\mathrm{Au}_{\mathrm{n}}$ nanoparticles formed on the surface. Spectroscopy set points: $1.5 \mathrm{~V}, 50 \mathrm{pA}$. Amplitude of bias modulation used: $20 \mathrm{mV}$. 

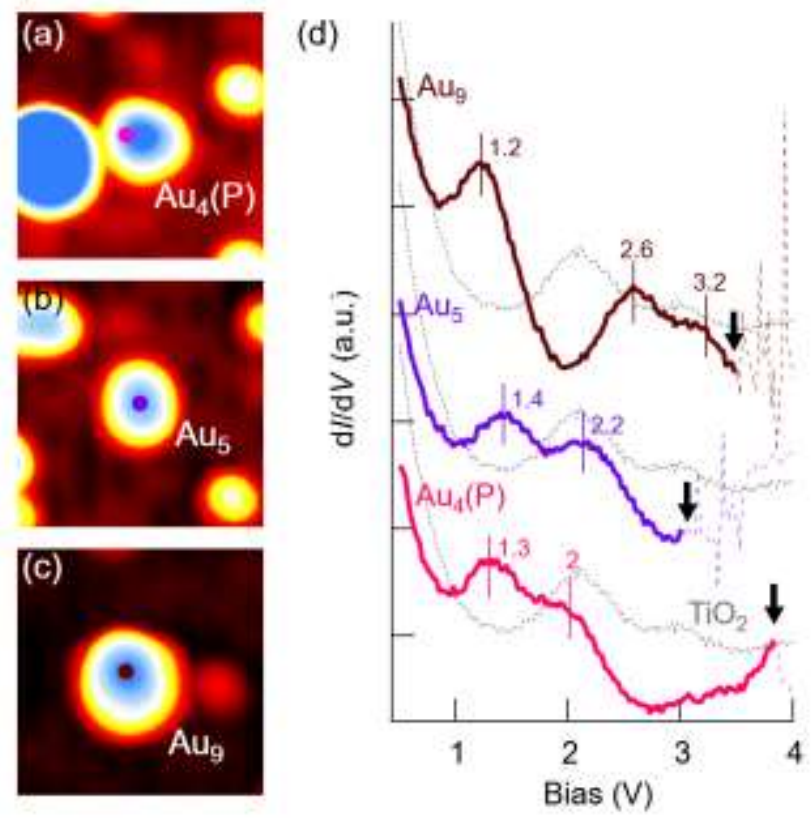

Figure S6. (a-c) STM images of (a) Au4(P) (where 'P' stands for 'planar' configuration), (b) Aus, and (c) Aug clusters. Scan parameters (V, I): (a,c) $1.1 \mathrm{~V}, 0.1 \mathrm{nA}$, (b) $0.8 \mathrm{~V}, 20 \mathrm{pA}$. (d) cc-dI/dV spectra $\left(I_{\mathrm{s}}=20 \mathrm{pA}\right)$ of the $\mathrm{Au}_{4}(\mathrm{P})$ (pink), $\mathrm{Au}_{5}$ (purple), and $\mathrm{Au}_{9}$ (brown) clusters. Dots in (a-c) mark the locations at which the spectra were taken. Black arrows indicate the moment where $\mathrm{Au}$ clusters were displaced by the tip at high bias voltages. The spectrum of $\mathrm{r}-\mathrm{TiO}_{2}(110)$ is overlaid on top of each $\mathrm{Au}_{\mathrm{n}}$ spectrum for visual guidance. Spectra are offset vertically for clarity. 


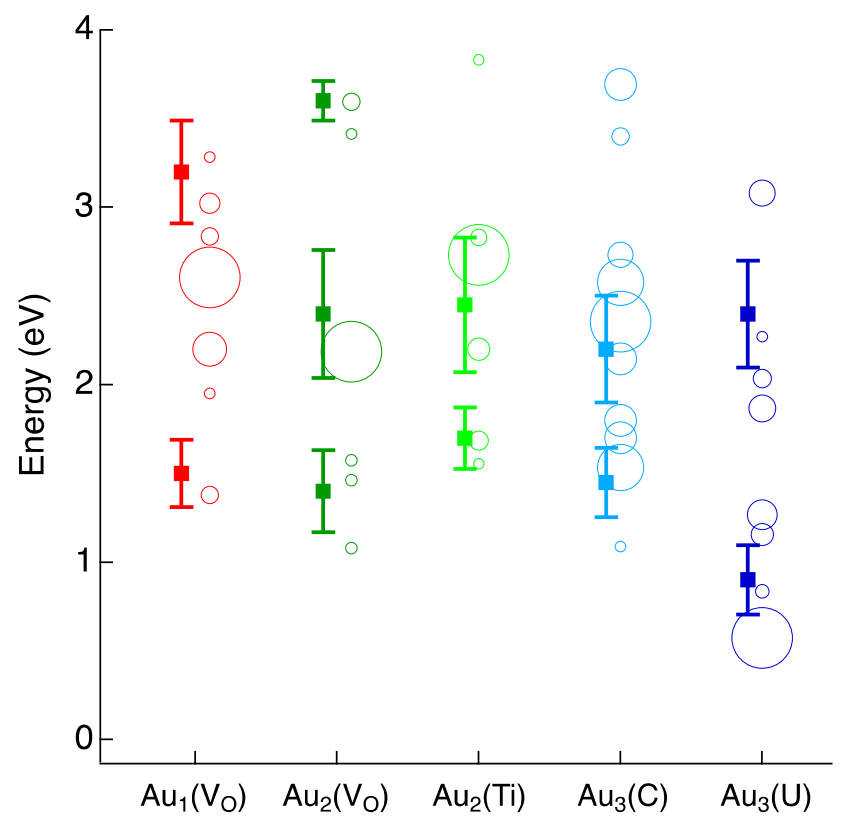

Figure S7. Scatter plot of the energy positions of the unfilled Au states for different $A u_{n}$ clusters on the $\mathrm{TiO}_{2}(110)$ surface. Solid squares are the bias voltage positions of the unfilled states peaks found in tunneling spectroscopy. Error bars denote the FWHM of each peak. The centers of the open circles denote the energy positions, relative to the $\mathrm{CBM}$ of bulk $\mathrm{TiO}_{2}$, of the unfilled $\mathrm{Au}$ states predicted in our calculations. The sizes of the circles represent the relative DOS of the unfilled Au states, normalized by that of the highest DOS for each Au cluster. The energy zero for the DOS is taken as the conduction band minimum.

\section{Supporting References}

(1) Kresse, G.; Hafner, J. Ab Initio Molecular Dynamics for Liquid Metals. Phys. Rev. B 1993, 47 (1), 558-561.

(2) Kresse, G.; Hafner, J. Ab Initio Molecular-Dynamics Simulation of the Liquid-MetalAmorphous-Semiconductor Transition in Germanium. Phys. Rev. B 1994, 49 (20), 1425114269.

(3) Kresse, G.; Furthmüller, J. Efficiency of Ab-Initio Total Energy Calculations for Metals and Semiconductors Using a Plane-Wave Basis Set. Comp. Mater. Sci. 1996, 6 (1), 15-50. 
(4) Kresse, G.; Furthmüller, J. Efficient Iterative Schemes for Ab Initio Total-Energy Calculations Using a Plane-Wave Basis Set. Phys. Rev. B 1996, 54 (16), 11169-11186.

(5) Perdew, J. P.; Burke, K.; Ernzerhof, M. Generalized Gradient Approximation Made Simple. Phys. Rev. Lett. 1996, 77 (18), 3865-3868.

(6) Perdew, J. P.; Burke, K.; Ernzerhof, M. Generalized Gradient Approximation Made Simple [Phys. Rev. Lett. 77, 3865 (1996)]. Phys. Rev. Lett. 1997, 78 (7), 1396-1396.

(7) Blöchl, P. E. Projector Augmented-Wave Method. Phys. Rev. B 1994, 50 (24), 1795317979.

(8) Kresse, G.; Joubert, D. From Ultrasoft Pseudopotentials to the Projector Augmented-Wave Method. Phys. Rev. B 1999, 59 (3), 1758-1775.

(9) Davidson, E. R. Matrix Eigenvector Methods. In Methods in Computational Molecular Physics; Springer, Dordrecht, 1983; pp 95-113.

(10) Dudarev, S. L.; Botton, G. A.; Savrasov, S. Y.; Humphreys, C. J.; Sutton, A. P. ElectronEnergy-Loss Spectra and the Structural Stability of Nickel Oxide: An LSDA+U Study. Phys. Rev. B 1998, 57 (3), 1505-1509.

(11) Stoneham, A. M. Systematics of Metal-Insulator Interfacial Energies: A New Rule for Wetfing and Strong Catalyst-Support Interactions. Appl. Surf. Sci. 1982, 14, 249-259.

(12) Puigdollers, A. R.; Schlexer, P.; Pacchioni, G. Gold and Silver Clusters on $\mathrm{TiO}_{2}$ and $\mathrm{ZrO}_{2}$ (101) Surfaces: Role of Dispersion Forces. J. Phys. Chem. C 2015, 119 (27), 15381-15389.

(13) Grimme, S. Semiempirical GGA-Type Density Functional Constructed with a Long-Range Dispersion Correction. J. Comput. Chem. 2006, 27 (15), 1787-1799.

(14) Tosoni, S.; Sauer, J. Accurate Quantum Chemical Energies for the Interaction of Hydrocarbons with Oxide Surfaces: $\mathrm{CH}_{4} / \mathrm{MgO}(001)$. Phys. Chem. Chem. Phys. 2010, 12 (42), 14330.

(15) Howard, C. J.; Sabine, T. M.; Dickson, F. Structural and Thermal Parameters for Rutile and Anatase. Acta Cryst. B 1991, 47 (4), 462-468.

(16) Tang, W.; Sanville, E.; Henkelman, G. A Grid-Based Bader Analysis Algorithm without Lattice Bias. J. Phys.: Condens. Matter 2009, 21 (8), 084204.

(17) Sanville, E.; Kenny, S. D.; Smith, R.; Henkelman, G. Improved Grid-Based Algorithm for Bader Charge Allocation. J. Comput. Chem. 2007, 28 (5), 899-908.

(18) Henkelman, G.; Arnaldsson, A.; Jónsson, H. A Fast and Robust Algorithm for Bader Decomposition of Charge Density. Comp. Mater. Sci. 2006, 36 (3), 354-360.

(19) Tersoff, J.; Hamann, D. R. Theory of the Scanning Tunneling Microscope. Phys. Rev. B 1985, 31 (2), 805-813. 